$J p n . J$. Genet. (1988) 63, pp. 67-73

\title{
Popuration genetics of cultivated common buckwheat, Fagopyrum esculentum Moench VI. The amount of detrimental mutant genes concealed in a population is the same in all local races in the world
}

\author{
Ohmi OHNISHI \\ Laboratory of Genetics, Faculty of Agriculture, \\ Kyoto University, Kyoto 606 \\ (Received October 23, 1987)
}

\begin{abstract}
The amount of chlorophyll-deficient and other detrimental recessive mutants concealed in a cultivated buckwheat population was estimated for 17 populations from different parts of the world, by conducting more than 3000 sib crosses. The following types of abnormalities were found in cotyledons; albino $0.2 \%$, yellow $3.4 \%$, pale yellow $5.2 \%$, pale green $7.5 \%$, variegated $1.0 \%$, morphological $3.5 \%$ and others $2.0 \%$ on the average. The frequency of each type did not vary so much among the populations and it coincided well with the frequency previously observed in the Japanese populations. Each type was also found in the foliage leaves at about $2 / 3$ of the frequencies in cotyledons. No case of polymorphism of chlorophyll-deficient or other detrimental mutants has been found so far. These data confirmed the conclusions given by Ohnishi (1982); most of the detrimental mutants appear to be maintained in a population at very low frequency by mutation-selection balance; cases of balanced polymorphism are extremely rare, if they exist at all, in cultivated buckwheat populations.
\end{abstract}

\section{INTRODUCTION}

Two or three decades ago, an assessment of the frequency of recessive lethals and other detrimental genes and the elucidation of their place in the genetic variability of a population were major problems in population genetics; at that time, these topics were extensively investigated, mostly with Drosophila species. Several cases of polymorphism for chlorophyll-deficient mutants in plants were also reported (Apirion and Zohary 1961, Curran 1963 and others; see the reviews by Crumpacker 1967, Solbrig 1980). However, it still remains to be seen if these are special cases, since data on the frequencies of these mutant alleles are relatively scarce. Sprague and Schuler (1961) reported that almost all mutants they studied in maize were present at low frequencies. This suggested that mutation-selection balance was the mechanism maintaining these detrimental mutants.

In the first three reports of the present series of studies on common buckwheat populations, the total amount of concealed mutants affecting chlorophyll- 
deficiencies, dwarfism and sterility was assessed by analyzing more than 3000 full-sib families in 18 Japanese populations (Ohnishi 1982, Ohnishi and Nagakubo 1982, Ohnishi 1985). In spite of the large number of sib-lines investigated, no case of polymorphism for chlorophyll-deficient or other types of detrimental mutant alleles was found. Based on the estimated total frequency of detrimental mutant alleles per gamete and the frequency of homozygotes in randomly mating populations, the numbers of loci affecting chlorophyll-deficiencies, dwarfism and sterilities were estimated. More than 100 loci appeared to be involved and the mutant frequency at each locus was around 0.001-0.004; these results are consistent with those of Sprague and Schuler (1961).

Most of the cultivated common buckwheat in the world consists of local races; the cultivation of newly bred varieties is quite rare. Each buckwheat population is randomly mating because of complete outcrossing due to heterostyly. Furthermore, populations are always very large, usually consisting of more than $10^{4}$ plants. We should therefore expect the amount of detrimental mutant alleles concealed in a population to be the same in all populations, if these alleles are maintained by mutation-selection balance.

In the present study, 17 populations from different parts of the world were analyzed for the amount of detrimental genes affecting seedlings, mostly those affecting cotyledons and foliage leaves, by using the same analyses used by Ohnishi $(1979,1982)$. The total amount of concealed detrimental mutants in a population was the same in all populations and no cases of polymorphism were observed; these results suggest that mutation-selection balance is responsible for maintaining detrimental mutations in buckwheat populations.

\section{MATERIALS AND METHODS}

Common buchwheat populations analyzed in the present study were those listed in Table 1. Most of them were local land races in different countries; Hruszowska and Jubilejna were the commonly cultivated varieties in Poland and the Soviet Union, respectively. These materials were collected by buckwheat scientists and provided to me. I would like to express my sincere appreciation to Dr. Komenda for the Russian and Polish varieties, to Dr. Kreft for the Yugoslavian, French and Italian samples, to Drs. Ryu and Wang for the northern Chinese samples, to Dr. Huh for the Korean samples and to Dr. Gohil for the Kashimirian sample.

Starting from random seed samples of a population, approximately one to several hundred sib lines, each consisting of more than 30 seeds, were established by ordinaly sib-crosses (see Ohnishi 1979, 1982 for more details). Each line was investigated for the segregation of chlorophyll-deficient and other detrimental mutants at the seedling stage by sowing the $\mathrm{F}_{2}$ sibs in soil in a bed or in an experimental field. In this study, as in Ohnishi $(1979,1982)$, only the recessive mutants, that is, those that segregated according to a $3: 1$ ratio in the $F_{2}$ 


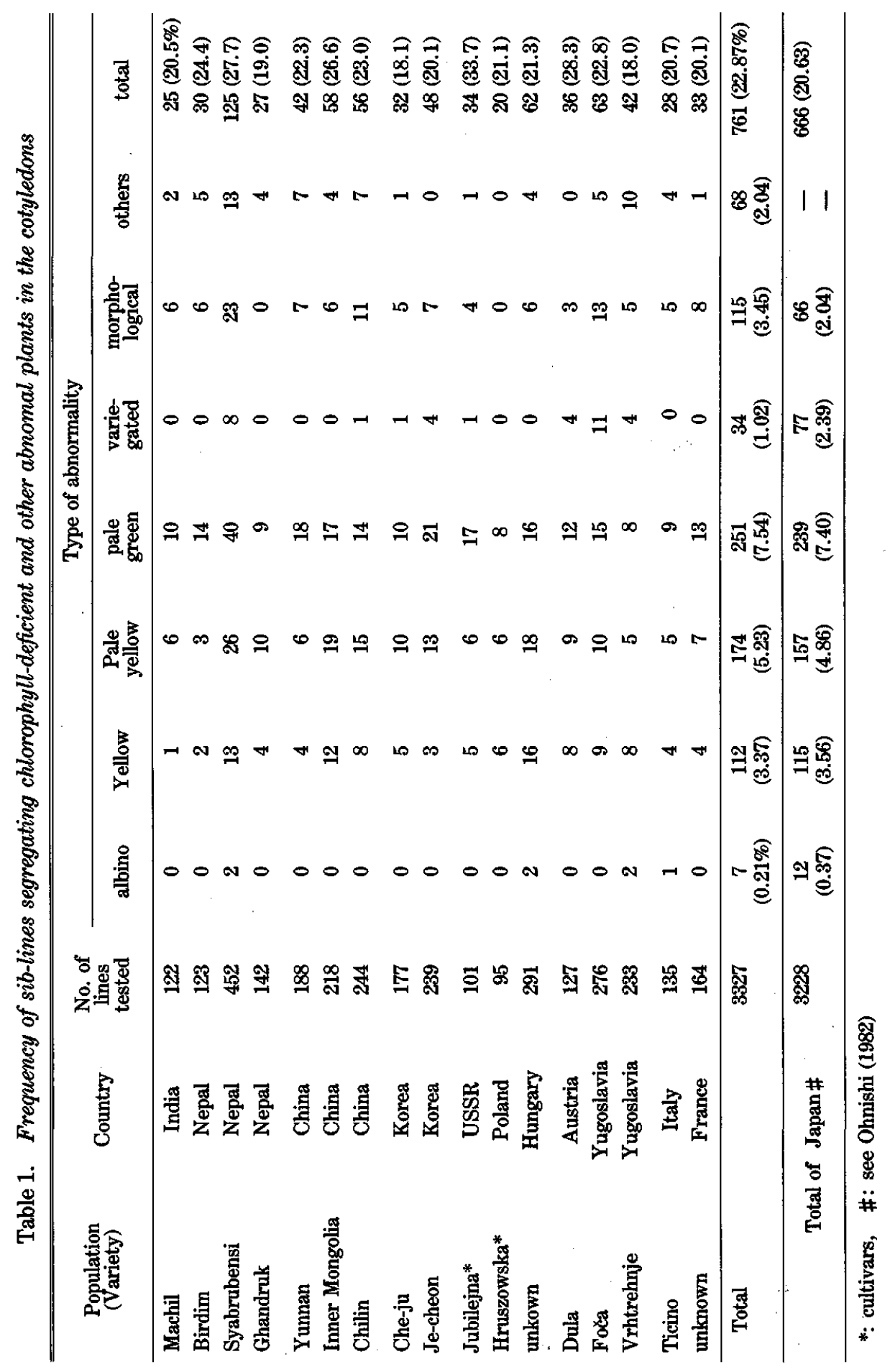


generation, were scored; those that segregated less frequently, such as 15:1, were deleted from the data by testing the observed numbers for a fit to the $3: 1$ ratio by the $\chi^{2}$ test. The frequency of sib-lines that segregated recessive homozygotes gives an estimate of the total frequency of recessive mutants per gamete as explained in Ohnishi (1979).

The mutants are classified into seven categories (see Table 1), primarily according to the leaf color of mutant plants. The classifications were rather arbitrally, but they were chosen so that the data were comparable with similar data in maize (see a photograph in Ohnishi 1979 and compare with the classification in Crumpacker 1967).

\section{RESULTS}

The numbers of sib-lines that segregated each category of chlorophyll-deficient and other detrimental genes affecting cotyledons are given in Table 1. In total, the following abnormalities were observed; albino only $0.2 \%$, yellow $3.4 \%$, pale yellow $5.2 \%$, pale green $7.5 \%$, variegated $1.0 \%$, morphological mutants including dwarf mutants $3.5 \%$ and others such as stem color mutants $2.0 \%$. These frequencies coincide well with those observed in the Japanese populations that are listed at the bottom of the table to facilitate comparison. An important observation was that the frequency of sib-lines that segregated recessive homozygotes did not vary much among populations from different parts of the world; it was lowest(18\%) in Vrhtrehnje, Yugoslavia and highest(34\%) in Jubilejna, a Russian variety.

Table 2 shows similar frequencies for the mutants affecting foliage leaves. The following frequencies were observed; albino $0.15 \%$, pale yellow $3.5 \%$, pale green $5.0 \%$, variegated $2.1 \%$, morphological $3.5 \%$ and others $0.8 \%$. Again the frequency of each category was quite similar with that observed in the Japanese populations. The values in the parentheses are the number of lines in which homozygous segregants with abnormal foliage leaves also suffered from a cotyledon abnormality. Some mutants affected only foliage leaves, but some affected both. The total frequency of segregant lines slightly varied among the populations. However, a careful observation of the table reveals that the frequencies of the variegated and morphological abnormalities were quite variable and these led to apparent differences among the populations.

\section{DISCUSSION}

First of all, it should be noted that in the present study, only recessive mutants affecting the seedling stage were investigated; other mutants that segregated less frequently were ignored.

The total amount of detrimental mutants maintained in each population was 


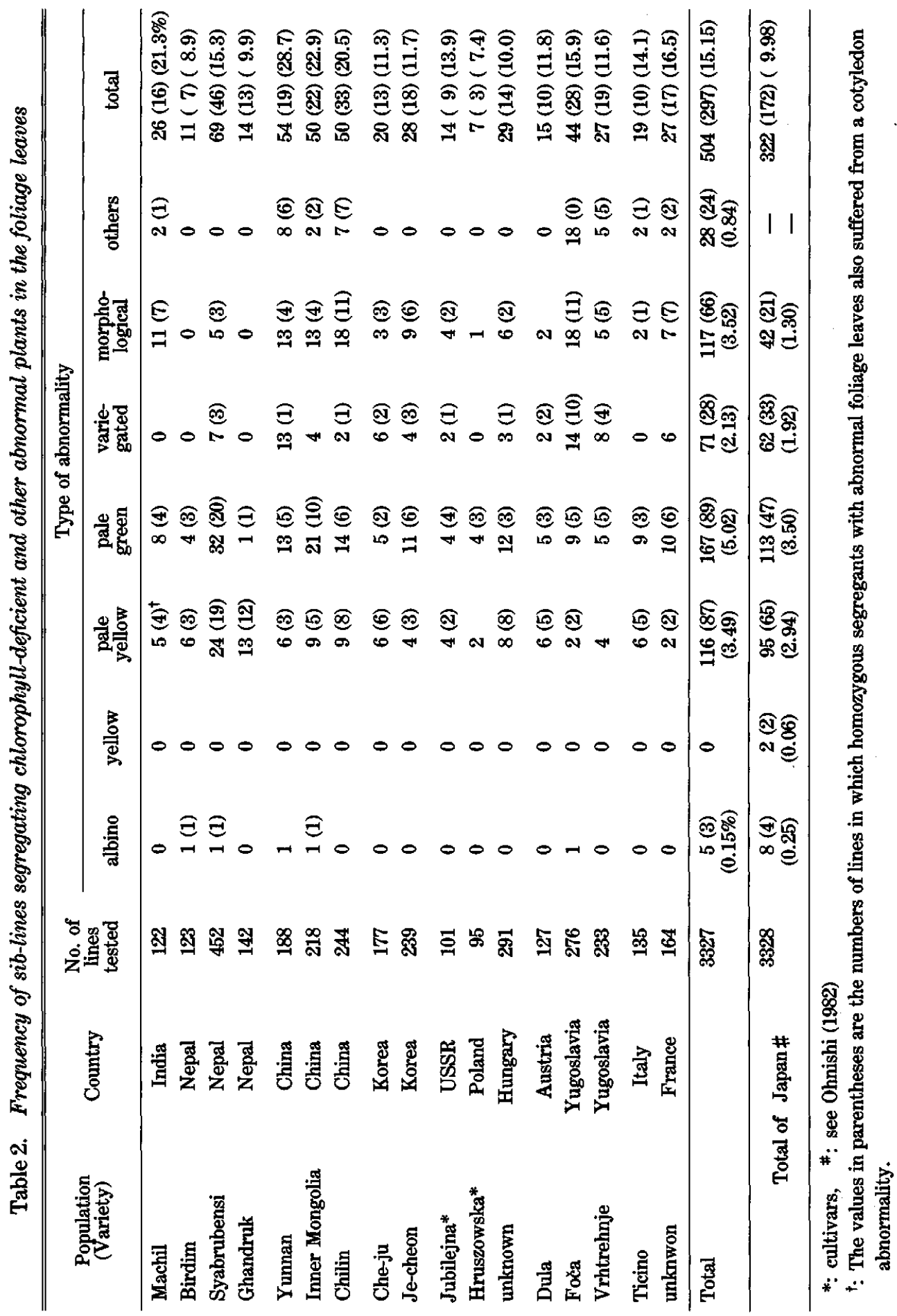


apparently the same; the homogenity of the frequency of each category between the populations was confirmed by the $\chi^{2}$ test. A similar conclusion was reached for the pale yellow and pale green mutants affecting foliage leaves.

Now what can we say about the total amount of detrimental genes maintained in a population and the mechanisms that maintain them? In the present study, the gene frequency at each locus was not directly estimated (cf. Sprague and Schuler 1961, Apirion and Zohary 1961, Ohnishi and Nagakubo 1982); neither was the incidence of each mutant homozygote in the randomly mating population observed. Hence, I cannot follow arguments similar to those of Ohnishi (1982). However, it is now apparent that the frequency of sib-lines that segregate mutant homozygotes, which gives an estimate of the total frequency of such mutants per gamete, is the same in all cultivated buckwheat populations in the world, including the Japanese populations. This is expected if each population is large and mating randomly with a long enough history to reach equilibrium and that the mechanism that maintains these mutants is the same in all populations. All the populations of buckwheat are large, usually consisting of more than $10^{4}$ individuals, and mating is random because of insect pollination and the random mixing of the seeds at harvet and sowing.

Therefore, I do not hesitate to adopt estimates for the number of loci and the frequency of individual mutant alleles that were derived from the studies of Japanese populations (Ohnishi 1982); thus, the number of loci and the frequency at each locus are 3.5 and 0.001 respectively for albino, 10 and 0.0035 for yellow, 26 and 0.0019 for pale yellow, 59 and 0.0013 for pale green, and 7.5 and 0.0032 for variegated. These estimates explain well the observed frequencies (Table 1) for the populations studied here. Similar estimates for the mutants affecting only foliage leaves (see Table 6 of Ohnishi 1982) explain the frequencies in Table 2 in this study.

So far as detrimental mutants are concerned, cases of polymorphism have not been observed in common buckwheat. A possible unique exception is the mutant causing a determinate form of inflorescence found by Kreft (1983). It is segregating at a high frequency in the Ticino population in Italy and also in several populations in Yugoslavia in very low frequency (Ohnishi unpublished); but the mechanisms that maintain it have not been studied so far.

As a conclusion to the first part of this series of studies on cultivated common buckwheat, I can say that almost all detrimental mutants are maintained at a low frequency, probably by mutation-selection balance. The number of such mutant loci is large, more than a hundred, and the mutant frequency at each locus is around 0.001-0.004; therefore the total amount of detrimental mutants per gamete which is equal to the observed frequency of sib-lines segregating such mutants, approaches 20-30\%. Direct estimates of the frequencies of detrimental mutants are still scarce in studies of plant populations, but Sprague and Schuler (1961) and Ohnishi and Nagakubo (1982) arrived at the same conclusion for a low 
frequency at each locus. Cases of balanced polymorphisms of chlorophylldeficient and other detrimental mutants in plant populations are probably quite rare. Occasionally there are cases where morphological mutant alleles are polymorphic; but in these, the role of selection is entirely obscure.

I am grateful to Dr. Michael J. Simmons, the University of Minnesota, for his reading manuscript, correcting English and making numerous useful suggestions.

\section{REFERENCES}

APIRION, D. and ZoharY, D. (1961) Chlorophyll lethal in natural populations of the orchard grass (Dactylis glomerata L.) A case of balanced polymorphis in plants. Genetics 46, 393-399.

CrumpaCkER, D. W. (1967) Genetic loads in maize (Zea mays L.) and other cross-fertilized plants and animals. Evol. Biol. 1, 306-424.

Curran, P. L. (1963) Balanced polymorphisms in Datylis glomerata sub-species woronowii. Nature $197,105-106$.

KREFT, I. (1983) Buchwheat breeding perspectives. Buckwheat Research, Proc. 2nd Intl. Symp. Buckwheat 39-50.

OHNISH, O. (1979) Frequency of chlorophyll-deficient and other detrimantal genes in Japanese populations of buckwheat, Fagopyrum esculentum Moench. Jpn. J. Genet. 54, 259-270.

OHNisH, O. (1982) Population genetics of cultivated common buchwheat, Fagopyrum esculentum Moench. I. Frequency of chlorophyll-deficient mutants in Japanese populations. Jpn. J. Genet. 57, 623-639.

OHNISHI, O. (1985) Population genetics of cultivated common buckwheat, Fagopyrum esculentum Moench. III. Frequency of sterility mutants in Japanese populations. Jpn. J. Genet. 60, 391-404.

OHNishi, O. and NaGakubo, T. (1982) Population genetics of cultivated common buckwheat, Fagopyrum esculentum Moench. II. Frequency of dwarf mutants in Japanese populations. Jpn. J. Genet. $57,641-650$.

SolBRIG, O. T. (1980) Genetic structure of plant populations. In Demography and Evolution in Plant Population (ed. 0. T. Solbrig), pp. 49-65.

Sprague, G. and Schuler, J. (1961) The frequency of seed and seedling abnormalities in maize. Genetics 46, 1713-1720. 